\title{
Intravenous Ferric Carboxymaltose for Acute Isovolemic Anemia Following Gastrectomy (Fairy): A Randomized Controlled Trial
}

Young-Woo Kim ${ }^{1)}$, Jae-Moon Bae ${ }^{2)}$, Young-Kyu Park ${ }^{3)}$, Han-Kwang Yang ${ }^{4)}$, Wansik $\mathrm{Yu}^{5}$, Jeong Hwan $\mathrm{Yook}^{(6)}$, Sung Hoon $\mathrm{Noh}^{7)}$, Keun Won Ryu ${ }^{1)}$, Tae Sung Sohn ${ }^{2)}$, Hyuk -Joon Lee ${ }^{4)}$, Oh Kyoung Kwon ${ }^{5)}$, Seung Yeob Ryu ${ }^{3)}$, Jun-Ho Lee ${ }^{2)}$, Sung Kim ${ }^{2)}$, Hong Man Yoon ${ }^{1)}$, Bang Wol Eom ${ }^{1)}$, Min-Gew Choi ${ }^{2)}$, Beom Su Kim ${ }^{6}$, Oh Jeong ${ }^{3)}$, Yun-Suhk Suh ${ }^{4)}$, Moon-Won Yoo ${ }^{6)}$, In Seob Lee ${ }^{6)}$, Mi Ran Jung ${ }^{3)}$, Ji Yeong $\mathrm{An}^{2)}$, Hyoung-Il Kim ${ }^{7)}$, Young Sook Kim ${ }^{8)}$, Byung-Ho Nam ${ }^{9}$

1) Department of Cancer Control and Policy, Graduate School of Cancer Science and Policy, and Gastric Cancer Branch, Research Institute and Hospital, National Cancer Center

2) Center for Gastric Cancer, Samsung Medical Center, Sungkyunkwan University School of Medicine

3) Department of Gastroenterologic Surgery, Chonnam National University Hwasun Hospital

4) Department of Surgery, Seoul National University College of Medicine

5) Center for Gastric Cancer, Gastric Cancer Center, Kyungpook National University Medical Center

6) Center for Gastric Cancer, Asan Medical Center, University of Ulsan College of Medicine

7) Department of Gastrointestinal Surgery, Yonsei University Health System

8) Center for Gastric Cancer, Research Institute and Hospital, National Cancer Center

9) Cancer Registration \& Biostatistics Branch and Center for Clinical Trials, National Cancer Center

Aim : Acute isovolemic anemia is commonly observed after surgery and negatively influences shortand long-term outcomes. Current blood management practices fail to deliver effective reversal of anemia. The aim of this study was to evaluate the efficacy and safety of ferric carboxymaltose to treat anemia following gastrectomy.

Method: The FAIRY study was a patient-blind, randomized, phase 3, placebo-controlled, 12- week study was conducted between 4 February 2013 and 15 December 2015 to evaluate the ability of ferric carboxymaltose to correct acute isovolemic anemia. This study was conducted in seven centers across the Republic of Korea. The primary endpoint was the number of hemoglobin responders, defined as hemoglobin increase of $\geq 2 \mathrm{~g} / \mathrm{dL}$ from baseline and/or $\geq 11 \mathrm{~g} / \mathrm{dL}$ at week 12 . Secondary endpoints included changes in hemoglobin and other iron parameters over time, percentage of patients requiring alternative anemia management, and quality of life at weeks 3 and 12 .

Results : 454 patients were randomized to receive ferric carboxymaltose (228 patients) or placebo (226 patients). The number of hemoglobin responders was significantly greater for ferric carboxymaltose versus placebo (200 patients [92.2\%] vs 115 patients [54.0\%] ; $90 \mathrm{P}=0.001$ ). Correction of anemia and improvements in iron parameters were significantly in favor of ferric carboxymaltose at all time points, and these patients required less alternative anemia management compared to placebo patients (1.8\% vs $7.1 \% ; \mathrm{P}=0.006$ ). Improvements were observed for fatigue and dyspnea in the ferric carboxymaltose group. No grade 3 or 4 adverse events were recorded, and ferric carboxymlatose-related adverse events reported in more than one patient included injection site reaction and urticaria (both : 5 patients [2.3\%]) Conclusions : Ferric carboxymlatose for postoperative blood management was safe and effective in correcting anemia and iron deficiency. In order to improve the recovery period, patients with low hemoglobin levels post-gastrectomy should receive intravenous ferric carboxymaltose. 